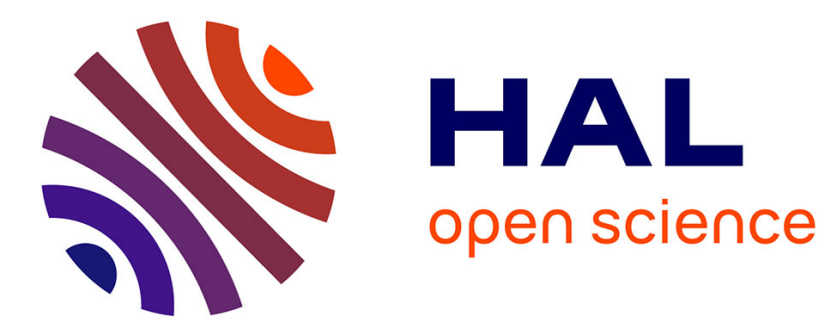

\title{
Du domicile à la ville: étapes et espaces de l'encadrement parental des pratiques urbaines des enfants
}

Clément Rivière

\section{To cite this version:}

Clément Rivière. Du domicile à la ville: étapes et espaces de l'encadrement parental des pratiques urbaines des enfants. Espaces et sociétés (Paris, France), 2017. hal-03183144

\section{HAL Id: hal-03183144 \\ https://hal.science/hal-03183144}

Submitted on 26 Mar 2021

HAL is a multi-disciplinary open access archive for the deposit and dissemination of scientific research documents, whether they are published or not. The documents may come from teaching and research institutions in France or abroad, or from public or private research centers.
L'archive ouverte pluridisciplinaire HAL, est destinée au dépôt et à la diffusion de documents scientifiques de niveau recherche, publiés ou non, émanant des établissements d'enseignement et de recherche français ou étrangers, des laboratoires publics ou privés. 
II

VARIA 


\title{
Du domicile à la ville : étapes et espaces de l'encadrement parental des pratiques urbaines des enfants
}

Clément Rivière

\begin{abstract}
$B_{\text {ien que les enfants soient dès leur plus jeune âge « bombardés d'instruc- }}$ tions » relatives à leurs déplacements (Lofland, 1973), la recherche en sciences sociales n'a fait preuve que d'un intérêt limité pour la socialisation des enfants à la vie urbaine, à l'exception notable des travaux conduits en psychologie environnementale (voir notamment Prezza et al., 2001). Le rôle que jouent les parents dans ce processus semble dans une certaine mesure être naturalisé en contexte de « domestication » de l'enfance (Holloway et Valentine, 2000), alors même qu'il apparaît en filigrane de recherches menées sur d'autres objets. De récents travaux ont néanmoins contribué à mettre en lumière ce travail régulateur et sa différenciation sociale et sexuée (Valentine, 2004 ; Authier et Lehman-Frisch, 2012), ainsi que les liens entretenus par l'autonomie urbaine des enfants avec les représentations de leurs parents (voir notamment Depeau, 2003 ; Granié, 2010).
\end{abstract}

Clément Rivière, maître de conférences en sociologie, chercheur au Ceries (EA 3589), université Lille 3-Charles de Gaulle

clement.riviere@univ-lille3.fr 
S'appuyant sur une enquête par entretiens conduite auprès de parents d'enfants âgés de 8 à 14 ans dans deux aires urbaines situées à la périphérie interne de Paris (France) et de Milan (Italie), cet article se donne pour objectif de distinguer de manière analytique les étapes qui jalonnent l'encadrement parental des pratiques urbaines des enfants dans deux métropoles européennes contemporaines, et d'en mettre en lumière les mécanismes de franchissement. Dans cette perspective, il aplanit délibérément les contrastes entre les expériences enfantines, liés notamment au rang occupé dans la fratrie, aux propriétés sociales des familles et au sexe des enfants. Si l'analyse de la différenciation des pratiques parentales se trouve au cœur de la thèse de doctorat en sociologie sur laquelle le propos s'appuie (Rivière, 2014), il s'agit ici de mettre au jour des mécanismes de portée générale, en vue de proposer une description idéale-typique des modalités de passage des enfants de l'espace privé à l'espace local, puis aux espaces publics les plus fréquentés. Du domicile à la ville, pour reprendre le titre d'un ouvrage qui, s'il ne s'intéressait pas à l'encadrement parental, en restitue efficacement le caractère progressif (Authier, 2001a).

Après avoir montré que le souci de protéger les enfants des accidents de la circulation et des inconnus permet de mieux saisir le rôle joué aux débuts de l'encadrement par le domicile, protégé de ces deux dangers, nous nous intéresserons aux mécanismes de construction de la réassurance des parents, d'abord dans le cadre de la fréquentation d'espaces que nous qualifierons de "protégés », puis au sein d'une aire de plus en plus éloignée du domicile. Alors que les enfants constituent un bon révélateur du rapport entretenu de manière plus générale par les adultes avec les espaces publics (Rivière, 2012), cette description des étapes de la prise d'autonomie urbaine nous conduira à proposer en conclusion une définition sociologique de la notion de « quartier».

\section{UNE ENQUÊTE PAR ENTRETIENS À PARIS ET MILAN}

Le choix d'étudier l'encadrement parental des pratiques urbaines des enfants par le biais d'une approche localisée et comparée a conduit à sélectionner deux contextes urbains caractérisés par l'hétérogénéité sociale et culturelle de la composition de leur population. Ceux-ci partagent un certain nombre de caractéristiques du point de vue de leur morphologie et de leur trajectoire de développement.

Situé au nord-est de Paris, le quadrilatère Villette-Belleville est délimité au nord-est par le boulevard périphérique, au sud-est par la rue de Belleville, au sud-ouest par le boulevard de La Villette et au nord-ouest par le bassin de La Villette et le canal de l'Ourcq. Son nom lui a été donné au regard de sa forme et de sa localisation, à cheval sur le territoire des deux anciennes communes de La Villette et de Belleville. Comptant 125393 habitants d'après le recensement de la population de 2008 (dont 10,4\% étaient âgés de 6 à 14 ans), il correspond 
à une partie significative du $\mathrm{XIX}^{\mathrm{e}}$ arrondissement (dont la population s'élevait à 184909 habitants la même année).

Lui aussi situé au nord-est de Milan, le triangle Monza-Padova est délimité par deux voies de circulation importantes, Viale Monza à l'ouest et Via Palmanova à l'est. Nous lui avons toutefois donné le nom d'une autre voie de circulation qui le traverse, Via Padova, qui correspond à un axe historique du développement de la capitale lombarde. Son sommet est formé par le Piazzale Loreto, et sa base par la frontière entre Milan et Sesto San Giovanni, qui correspond pour une large part à des implantations industrielles désaffectées. Il comptait 76427 habitants au 31 décembre 2008 selon le bureau des statistiques de la ville (et 6,9\% d'enfants âgés de 5 à 14 ans d'après le recensement général de 2001).

Ces deux aires urbaines ont en commun d'avoir été des espaces agricoles et de villégiature jusqu'au XIX $X^{\mathrm{e}}$ siècle, puis d'avoir fait l'objet d'un processus d'industrialisation et de désindustrialisation. Leurs trajectoires parallèles éclairent l'hétérogénéité marquée de leur bâti, mais aussi celle de leur population : aucun des huit grands groupes socioprofessionnels distingués par l'Insee ne représentait plus d'un cinquième de la population active âgée de 15 ans et plus sur le terrain parisien en 2008, qui comptait la même année $15,7 \%$ d'étrangers. À Milan, le triangle Monza-Padova, terrain d'installation de migrants de l'intérieur durant plusieurs décennies, a été l'un des premiers de la ville à voir s'installer des migrants internationaux en nombre. Fin 2008, les étrangers comptaient pour près d'un quart des habitants (23,7\%), leur proportion ayant décuplé en moins de vingt ans. En lien avec sa désindustrialisation, sa composition sociale a également évolué au cours des dernières décennies : nombre d'ouvriers ont été remplacés par des employés et des chefs de petites entreprises, contribuant à la rapprocher de celle de l'ensemble de la ville de Milan (Arrigoni, 2010).

Le recours ponctuel à l'observation ethnographique a permis de constituer un corpus de notes d'observation focalisée et non-focalisée sur les deux terrains, mais c'est dans le cadre d'une campagne d'entretiens semi-directifs qu'a été accumulé le matériau principal de la recherche. Entre 2009 et 2012, 78 entretiens ont été conduits auprès de 88 parents $(51$ mères, 17 pères et 10 couples) d'au moins un enfant âgé de 8 à 14 ans. À Milan, 43 entretiens ont été conduits en italien ( 28 mères, 7 pères et 8 couples) tandis que 35 l'ont été en français à Paris ( 23 mères, 10 pères, 2 couples). Certains ont partiellement ou intégralement été réalisés en présence d'un ou de plusieurs des enfants concernés $(n=6)$ ce qui leur a permis d'intervenir de manière plus ou moins spontanée en cours de discussion.

D'une durée moyenne de 73 minutes (de 30 à 165 minutes), ces entretiens ont été intégralement retranscrits dans leur langue de réalisation, avant de faire l'objet d'une analyse thématique. Commun aux deux terrains d'enquête, le guide d'entretien s'articulait autour de trois volets. Le premier était consacré à 
la vie de quartier et au parcours résidentiel des enquêtés. Cour de l'entretien, le second se concentrait sur les pratiques urbaines des enfants et l'encadrement parental. Un dernier volet permettait d'introduire un questionnement plus large sur le rapport des parents à la vie urbaine et à la surveillance des espaces publics.

Tableau 1 - Modes d'entrée en contact avec les enquêtés

\begin{tabular}{|l|c|c|c|}
\cline { 2 - 4 } \multicolumn{1}{c|}{} & Milan & Paris & Ensemble \\
\hline Démarchage dans un espace public ou semi-public & 19 & 8 & $27(34,6 \%)$ \\
\hline Contact par un « informateur-relais » & 12 & 5 & $17(21,8 \%)$ \\
\hline $\begin{array}{l}\text { Contact direct ou indirect par une association } \\
\text { de parents d'élèves }\end{array}$ & 2 & 12 & $14(17,9 \%)$ \\
\hline Recommandation par un enquêté précédent & 7 & 4 & $11(14,1 \%)$ \\
\hline Contact par une connaissance commune & 2 & 6 & $8(10,3 \%)$ \\
\hline Autre & 1 & - & $1(1,3 \%)$ \\
\hline Total & 43 & 35 & $78(100 \%)$ \\
\hline
\end{tabular}

Pourcentages arrondis à $0,1 \%$ près

Les parents interrogés, qui présentent une importante diversité du point de vue des principales variables de l'analyse sociologique (âge, sexe, niveau d'éducation, profession, origine nationale), ont principalement été recrutés par le biais de quatre canaux : le démarchage direct dans des espaces publics ou semi-publics, la mise en contact par des informateurs-relais au sein d'associations locales, de centres sociaux ou de lieux de culte, la mobilisation de réseaux de parents d'élèves, et la recommandation par un enquêté précédent (voir tableau 1).

\section{AU DOMICILE À L'ABRI DES INCONNUS ET DES VÉHICULES MOTORISÉS}

S'inscrivant dans le cadre d'une représentation largement partagée d'une enfance vulnérable, liée à l'incompétence supposée des enfants à discerner le danger (Cahill, 1990), le sentiment d'un devoir de protection des risques de l'accident de la circulation et de l'enlèvement se trouve au cœur des préoccupations parentales (Pain, 2006). Le rappel de ces deux principales menaces perçues peut paraître trivial, et l'est d'ailleurs dans une certaine mesure pour les parents eux-mêmes tant un ensemble de recommandations leur semble aller de soi. L'enseignement aux enfants qu'il convient de se méfier à la fois des inconnus et des véhicules motorisés se trouve ainsi souvent décrit comme correspondant à de « grands classiques », aux « choses que tout le monde dit aux enfants ». C'est bien la raison pour laquelle insister sur ces deux menaces s'avère nécessaire en vue de comprendre l'encadrement parental, dans la 
mesure où elles le structurent en profondeur - quoique l'intensité de leur perception diminue avec l'avancée en âge des enfants.

La place centrale que tiennent les accidents de la circulation et l'entrée en contact avec un inconnu malveillant dans les représentations parentales du danger permet notamment de mieux saisir le rôle que joue le domicile de l'enfant dans la découverte autonome de la ville. Alors que de nombreuses recherches ont montré sa centralité accrue dans la vie quotidienne des enfants au cours des dernières décennies (Karsten et Van Vliet, 2006), le domicile constitue de fait une étape importante du processus de prise d'autonomie urbaine. À l'abri des inconnus et des véhicules motorisés, les enfants y sont la plupart du temps d'abord laissés seuls avant d'être autorisés à se déplacer sans leurs parents dans l'espace local. Tandis que d'autres recherches ont montré que les enfants eux-mêmes envisagent leur domicile comme un espace protégé (Harden, 2000), apprendre à rester seul chez soi dans le cadre d'expériences dont la durée tend à s'allonger à mesure de leur répétition constitue souvent une étape préliminaire à la fréquentation autonome de l'espace local.

Une fois l'enfant considéré comme apte à rester seul au domicile, lui en confier les clés marque une étape ultérieure et importante, qui consacre la reconnaissance de sa capacité au passage autonome de l'espace privé à l'espace local, et réciproquement de l'espace local à l'espace privé. «Avoir la clé » représente dans cette perspective un motif de fierté pour les enfants : cette marque de confiance ou son absence s'appuient en partie sur des considérations pratiques liées aux horaires de présence de référents plus âgés au domicile, mais aussi sur une évaluation de la capacité de l'enfant à assumer cette responsabilité. Une responsabilité jugée importante, dans la mesure où les clés sont susceptibles d'être perdues ou la maison mal fermée.

« On va sans doute commencer à lui donner les clés de la maison de temps en temps. Et cette étape-là sera sans doute plus importante. Parce qu'en tant que citadin, il est habitué à la circulation, à traverser la rue, dès la naissance tu n'as pas le choix, je ne dis pas que ça devient naturel parce qu'il faut toujours faire attention mais bon... Alors que d'après moi, avoir la responsabilité des clés de la maison... On lui fera un certain nombre de recommandations le moment venu. » (Commercial, Milan. Un fils de 10 ans)

« Pour eux c'est le début de la confiance que le parent leur donne, pour elle ça a été une conquête. Le fait que je lui donne les clés de la maison, pour elle ça a été une... Elle m'en a été reconnaissante, sans le formaliser verbalement, mais elle s'est sentie plus responsable. C'est un signe d'autonomie, c'est un signe de confiance, de confiance réciproque. » (Cadre d'entreprise, femme, Milan. Deux filles de 12 et 9 ans)

La centralité accrue du domicile et notamment de la chambre dans les expériences enfantines (voir notamment Glévarec, 2010) joue ainsi un rôle ambivalent du point de vue des pratiques urbaines des enfants. Si celle-ci 
favorise d'un côté le déclin de leur présence dans les espaces publics (Rivière, 2016), le domicile d'ego et des pairs constitue dans le même temps une étape importante dans l'accession à l'autonomie. Un tel constat invite à envisager le domicile comme un tremplin vers la ville, à l'image d'un ensemble d'autres espaces extérieurs mais considérés comme relativement sûrs par les parents.

\section{EN DEHORS DE CHEZ SOI, UNE GRADATION FINE D'ESPACES PERÇUS COMME PROTÉGÉS}

Hors du domicile, tout un ensemble d'espaces tels que certaines petites places ou rues piétonnes, des squares et des parcs, peuvent être considérés par les parents comme protégeant dans une certaine mesure leurs enfants des deux menaces décrites plus haut. Tout d'abord parce que la circulation motorisée y est interdite ${ }^{1}$, mais aussi car ils perçoivent que s'y exercent des formes de régulation des comportements. La perception des autres parents comme un « appui » (Femme au foyer, Paris, deux filles de 12 et 3 ans, deux fils de 11 et 8 ans) dans la surveillance de ses propres enfants, mais aussi des tiers, est ainsi largement partagée par les enquêtés, à l'image d'un père (enseignant dans une école privée, Paris, trois fils de 32, 11 et 7 ans, une fille de 7 ans) qui évoque de manière révélatrice une « responsabilité collective ». Alors que l'interconnaissance favorise la surveillance croisée et diffuse des enfants ainsi que leur réorientation lorsqu'ils s'égarent, certains parents mettent en œuvre un véritable contrôle des comportements des autres utilisateurs et veillent à la circulation de l'information relative à ces derniers, en particulier lorsqu'ils sont repérés comme menaçants ou érigés en menaces potentielles.

« On contrôle avec les autres parents que tout se passe bien, qu'il n'y ait pas de situations déplaisantes créées par des adolescents ou par des adultes, et on essaie de faire comprendre aux adultes que ce parc est [elle insiste] fréquenté par des enfants, et donc que chacun ne peut pas y faire tout ce qu'il voudrait. » (Employée à temps partiel dans un service après-vente, Milan. Une fille de 14 ans, un fils de 9 ans)

« Il y a quelques personnes que l'on a repérées qui sont un peu... Entre nous, entre mamans, on se dit ce genre de choses. "Il y a un monsieur, avec un chapeau, âgé, qui a ennuyé tel enfant...” " (Couturière, Milan. Une fille de 12 ans)

Les caractéristiques des espaces que les parents considèrent comme protégés (protection du risque d'accident de la circulation d'une part, régulation collective des comportements de l'autre) les érigent en arènes sécurisées

1. Sur le modèle des woornerfs néerlandais, la pacification de la circulation par des aménagements spécifiques et par la limitation drastique de la vitesse autorisée semble de manière plus générale favoriser les déplacements autonomes des enfants en milieu urbain (Huguenin-Richard, 2010). 
d'entraînement à l'autonomie, parfois explicitement envisagées en ce sens (voir également Prezza et al., 2001 ; Depeau 2003).

«Le Trotter [nom d'un grand parc du terrain milanais] permet à des enfants qui ont 8-9 ans de se balader seuls en toute tranquillité, parce qu'à l'intérieur ils ont leurs repères, et naturellement ensuite... Pour ma fille ça c'est vraiment passé comme ça, elle a d'abord commencé à gagner son autonomie à l'intérieur des limites du parc, et ensuite elle a peu à peu agrandi son périmètre. » (Chercheuse à l'université, Milan. Une fille de 10 ans, un fils de 9 ans)

Leur morphologie, leur équipement et le niveau d'interconnaissance perçue qui leur est associé différencient ces espaces le long d'une gradation fine. Ainsi, comme les stations de métro qu'ils fréquentent distinguent des « périodes » successives dans la vie des Parisiens (Augé, 1986), la fréquentation des espaces publics est scindée en diverses « périodes » dans l'expérience des parents. Des jeux considérés comme ne correspondant plus à leur âge par les enfants et leur réticence à fréquenter des espaces qu'ils associent à plus jeunes qu'eux mènent en effet à l'abandon progressif d'espaces pourtant assidûment fréquentés auparavant, le plus souvent au bénéfice d'autres espaces protégés. La demande des enfants qui grandissent n'est toutefois pas seule à entrer en compte, les espaces protégés se trouvant associés par les parents à des ambiances distinctes. Des parcs sont ainsi considérés comme plus « familiaux » que d'autres : on observe par exemple une opposition forte sur le terrain parisien entre le parc des Buttes-Chaumont et le parc de la Butte du ChapeauRouge, ce dernier étant présenté par de nombreux parents comme un lieu où l'on peut se retrouver le week-end sans avoir besoin de s'organiser à l'avance. Comme dans le cas des parties de football informelles qui se déroulent de manière régulière dans certains lieux ouverts à tous, un « common knowledge local » associe des lieux à des pratiques spécifiques (Trémoulinas, 2008).

«Alors, les Buttes-Chaumont, pourquoi on y va moins ? Parce que les jeux qu'il y a sont des jeux pour des enfants qui sont plus jeunes, ça les embête. Euh, je sais pas, les jeux sont enclavés donc du coup ils se sentent moins à l'aise, c'est plus petit, alors que dans le parc de la Butte-Rouge [nom donné localement au parc de la Butte du Chapeau-Rouge], d'abord il y a des gens plus âgés donc ils sont moins... Les enfants sont très sensibles aux enfants qu'ils fréquentent, ils ne veulent pas être pris pour des bébés. » (Chasseur de têtes pour un cabinet de recrutement, Paris. Un fils de 9 ans et une fille de 7 ans)

« Le parc des Buttes-Chaumont est très mélangé, les gens viennent d'autres quartiers. De la même manière que nous, les gens du XIX ${ }^{e}$, on pourrait aller au Jardin des plantes, vous voyez ? Alors que le parc de la Butte-Rouge reste ce que j'appelle un parc vraiment de quartier, là ce n'est pas un parc que les gens auront vu dans un guide touristique pour aller visiter. Alors que je suis sûre que le parc des Buttes-Chaumont y figure, lui. Vous voyez? Donc... La population est par conséquent différente. Au parc de la Butte-Rouge on retrouvera essentiellement les gens du quartier. » (Conseillère de vente à temps partiel, Paris. Trois fils de 13, 11 et 7 ans) 
La figure du « parc de quartier » mobilisée par cette mère renvoie à un niveau élevé d'interconnaissance perçue, producteur de confiance. Les squares et les petits parcs tendent ainsi à être considérés comme plus propices à leur fréquentation autonome par les enfants, tandis que les parents se montrent souvent plus réticents à l'idée de laisser leurs enfants évoluer en dehors de la présence d'un adulte dans les parcs les plus grands, que l'on pourrait qualifier par contraste de «parcs métropolitains ». Les parcs les plus grands sont toutefois le plus souvent eux-mêmes équipés d'espaces perçus comme davantage protégés, ces aires de jeu fermées invitant à penser la hiérarchisation du niveau de protection perçue au sein d'un même espace. C'est par exemple le cas du Jardin des dunes et des vents situé dans le parc parisien de La Villette, au sein duquel la fille de ces deux enquêtés peut évoluer librement alors qu'il lui est, pour l'instant, interdit de fréquenter le reste du parc sans adulte.

Père : Le parc de La Villette toute seule, elle n'y va pas, c'est hors de question. Chercheur : Pourquoi ?

Père : Parce que je trouve que... Elle n'a que 10 ans, et qu'à 10 ans, ben tu traînes pas toute une après-midi dans un parc tout seul, sans la responsabilité d'un adulte. Je sais pas, après je peux peut-être commencer à faire vieux jeu, mais moi c'est mon avis.

Chercheur : Donc elle y va des fois avec un de vous deux ?

Ensemble : Voilà !

Mère : On fait pas mal de trucs ensemble aussi.

Père : Par exemple, des fois elle vient me voir : « Ouais, on va au parc, on y va avec la mère ou avec la grande sœur d'une des copines ». Bon, okay.

Mère : Ouais, et puis souvent ils vont au Jardin des vents, donc c'est fermé aussi, et voilà, c'est pas comme être dans le parc avec plein de gens autour.

(Technicien son et lumières, technicienne de laboratoire, Paris. Une fille de 10 ans)

Parallèlement à cette hiérarchisation du niveau de protection associé aux différents espaces, qui peut donc varier à l'intérieur de chacun d'entre eux, le niveau d'interconnaissance perçue évolue pour un même espace en fonction des moments de la journée, de la semaine ou de l'année. Les espaces considérés comme " protégés » le sont donc avant tout en lien avec la quantité et surtout la qualité perçues de leur fréquentation.

\section{LES RESSOURCES DE L'ENCADREMENT DANS L'ESPACE LOCAL}

En dehors de ces espaces perçus comme protégés par les parents, les premières expériences de mobilité autonome des enfants dans l'espace local se déroulent le plus souvent au sein d'un périmètre restreint aux abords du logement. De petites commissions, par exemple à la boulangerie ou à l'épicerie la plus proche, correspondent ainsi presque toujours à la première expérience d'autonomie. De courts trajets vers ou depuis une activité extrascolaire ou 
encore vers le domicile d'un ami ou d'une amie résidant à proximité participent également de ces premières formes de mobilité autonome dans l'espace local, parfois supervisées depuis une fenêtre ou un balcon par l'un des parents.

Ce petit périmètre tend à s'élargir progressivement, les parents s'appuyant notamment sur les commerces pour encadrer cette découverte de l'espace local. Considérés comme autant de refuges potentiels et érigés en ressources protectrices (voir également Depeau, 2003, 2008), ceux-ci sont généralement présentés par les parents à leurs enfants comme des espaces sécurisés, où il est possible de demander son chemin ou un renseignement, ou encore d'essayer d'entrer en contact avec sa famille. Les commerçants sont par ailleurs considérés comme susceptibles d'intervenir de leur propre chef en cas de difficulté, en particulier lorsqu'ils connaissent l'enfant : la confiance que les parents tendent à leur accorder contribue de manière significative à la production d'un sentiment de sécurisation de la mobilité des enfants dans l'espace local.

«Voilà ce que je dis à mes enfants : "Si vous voyez que vous êtes en difficulté, que quelqu'un vous suit, et que vous ne savez pas quoi faire, entrez dans un bar, entrez dans un commerce et dites : "Cette personne me suit, j'ai peur et je voudrais appeler ma maman" ». (Femme au foyer, Milan. Trois fils de 16, 13 et 8 ans)

« Je leur ai toujours dit que si un jour dans le quartier, ou même dans la rue, on se faisait embêter par un adulte, de rentrer dans un commerce. Une pharmacie ou un commerce. Et expliquer ce qui leur arrive. C'est le b.a.-ba, quoi, on va dire, de la survie, de la survie à Paris. » (Employée des postes, Paris. Deux fils de 13 et 11 ans, une fille de 8 ans)

Au-delà de ce rôle de réassurance joué par la figure du commerçant, la sérénité dont font preuve les parents vis-à-vis de la fréquentation diurne de l'espace local par leurs enfants s'appuie aussi sur la confiance qu'ils manifestent vis-à-vis de l'exercice d'une surveillance diffuse par d'autres utilisateurs des espaces publics. Les propos de cette mère de famille aisée, élevée dans un quartier bourgeois de l'ouest de Paris, sont particulièrement révélateurs. Guère à l'aise à son arrivée vis-à-vis des jeunes hommes qui se regroupent en « bandes » dans les rues adjacentes à son logement, elle les considère désormais dans une certaine mesure comme des acteurs de la sécurisation de l'espace local pour ses enfants :

" Même les bandes qui sont dites "racailles", qui stagnent devant les terrains de basket, quand on a un petit qui se casse la figure, et ben les ados vont être les premiers à se précipiter, en me faisant la morale même : "Attention, il tombe, ça glisse, il faut lui donner la main !" Je veux dire, l'être humain, la plupart du temps, aide. Et ceux dont $\mathrm{j}$ 'avais peur en arrivant dans le quartier, plein de fois ils ont ramassé l'enfant qui se cassait la figure. Ce sont souvent des aînés de fratrie, ils vont être attentifs aux petits. Alors, on va les voir se battre, ils vont effrayer tout le monde, mais s'il y a un enfant qui va traverser en courant, ils vont lui faire la morale. [...] Et moi ils vont presque me rassurer. À la fois m'angoisser parce 
qu'ils sont toujours... Ils se battent, ils se battent en fait pour rire, mais ils se battent, et donc ça fait peur, les grands gaillards qui se tapent dessus, mais je sais qu'ils sont vigilants aussi. » (Femme au foyer, Paris. Deux filles de 12 et 3 ans, deux fils de 11 et 8 ans)

Les entretiens conduisent ainsi à remettre en cause la thèse du déclin des " yeux de la rue » (eyes on the street). Forgé par Jane Jacobs, ce concept insiste sur le rôle joué dans le maintien de la « paix publique » par un ensemble de contrôles informels, mis en œuvre de manière plus ou moins consciente par les individus qui habitent ou fréquentent l'espace local (Jacobs, 1961). Mais si pour Jacobs le fonctionnement des « yeux de la rue » ne requiert pas nécessairement l'interconnaissance, la confiance dans la supervision collective de la mobilité des enfants dans l'espace local se trouve renforcée par le sentiment de son existence, liée notamment à l'expérience de la coprésence résidentielle. Correspondant souvent à des « liens faibles » (Granovetter, 1973), l'interconnaissance locale, que l'on pourrait également qualifier d'inter-reconnaissance (Simon, 1997), renforce la confiance dont les parents font preuve vis-à-vis du fonctionnement de mécanismes de supervision collective et informelle des pratiques urbaines enfantines (voir également Depeau, 2008).

Cette confiance relativement élevée qu'ont les parents envers la surveillance diffuse de la mobilité des enfants repose en partie sur leur propre propension à la vigilance et à l'interventionnisme. Deux dimensions doivent ici être distinguées : la prévention du risque d'enlèvement ou d'agression d'une part, la répression des comportements dangereux de l'enfant lui-même ou de son groupe de pairs de l'autre. Un tel constat invite à considérer les parents à la fois comme des « consommateurs » et comme des «producteurs » des ressources disponibles dans l'espace local (Galster, 2001) : leur niveau de confiance dans la sécurité des enfants s'appuie de fait sur des attentes (expectations) relatives à l'engagement réciproque d'adultes dans la supervision des pratiques des enfants (Sampson et al., 1999).

« Non, moi je ne pense pas par exemple qu'une petite fille puisse être embêtée sur ce trajet où il y a plein d'autres parents avec des enfants. Je pense que si ça arrivait, même si des plus grands l'embêtaient, je pense que, enfin j'imagine que des parents interviendraient toujours. Tout de suite. Et moi je le ferais ! » (Maître de conférences à l'université, femme, Paris. Deux fils de 13 et 1 an, une fille de 9 ans)

Cette confiance dans la supervision collective de la mobilité des enfants dans l'espace local, qui repose en partie sur des normes urbaines à la validité plus générale, s'appuie en particulier sur l'hypothèse que les autres parents sont autant de street watchers (Jacobs, 1961) potentiels. Elle varie avec le niveau perçu d'interconnaissance, que la fréquentation régulière des commerces et des espaces « protégés » de l'espace de résidence contribue à renforcer. 


\section{ACCOMPAGNER LA DÉCOUVERTE DE LA GRANDE VILLE}

Symboles de la spécificité des comportements humains en milieu urbain (Simmel, 1992), les transports en commun ne font en aucun cas partie des espaces que les parents considèrent comme protégés. L'apprentissage de leur utilisation fait au contraire l'objet d'un ensemble de précautions spécifiques, les premiers trajets effectués en autonomie étant dans la quasi-totalité des cas des trajets brefs et sans correspondance. Ils peuvent par ailleurs être précédés d'une période d'accompagnement, qui peut correspondre au seul premier trajet ou se prolonger dans le temps.

« Pour aller, par exemple, à Piazza Argentina, vers la gare centrale, il n'y va pas tout seul. Je l'accompagne. Parce que ce trajet implique un changement un peu compliqué à l'intérieur du réseau, le passage de la ligne rouge à la ligne verte, ce qu'il n'a pas l'habitude de faire. Je ne suis pas sûre qu'il soit en mesure d'y arriver tout seul. » (Cadre de banque, femme, Milan. Deux fils de 12 et 8 ans)

Un ensemble de recommandations entourent l'apprentissage de l'utilisation des transports en commun. Il s'agit pour les parents de transmettre à la fois des connaissances et des manières d'agir : apprendre à bien valider son ticket et à ne pas frauder, connaître les lignes et leurs arrêts ainsi que les correspondances et surtout ne pas se tromper de sens au moment de monter dans le métro ou le bus. L'imprévu cristallise leurs préoccupations, qu'il s'agisse de situations d'interaction avec d'autres voyageurs auxquelles les enfants ne seraient pas préparés - comme les musiciens ambulants ou les sans-abri - ou bien de problèmes techniques ou d'inattention qui les conduiraient à devoir descendre à un arrêt inconnu.

«La ville c'est plein d'embûches, Paris c'est plein d'embûches, quand vous prenez le métro, il suffit qu'il y ait une panne, on vous dit de descendre, c'est la panique, quoi. [...] Tout d'un coup, qu'est-ce que vous faites, vous êtes descendu, mais ne serait-ce qu'une station plus loin, vous descendez à Riquet au lieu de Crimée, si elle descend à Riquet elle va être perdue, elle n'a pas encore de repères... Tout en habitant dans le quartier depuis dix ans, elle ne va pas s'y retrouver, elle va être perdue. Donc il faut quand même bien les guider un minimum. [...] Un truc tout bête, oui, le métro qui finalement a une panne, on leur dit qu'il faut descendre, qu'est-ce qui se passe ? Tant qu'on ne sait pas réagir à ça on ne peut pas partir toute seule, c'est pas possible. » (Experte-comptable, Paris. Deux filles de 13 et 10 ans, un fils de 7 ans)

L'accompagnement par les parents peut être l'occasion d'expérimenter une semi-autonomie : suivre à distance l'enfant dans le métro permet notamment d'observer et d'évaluer sa capacité à le prendre seul. Les entretiens contiennent de multiples exemples de ces mises à l'épreuve, couronnées d'un succès variable. 
« Au printemps dernier, je les ai emmenées au Trocadéro avec des copines faire du roller, et je leur ai dit: “C'est vous qui allez me guider, je vous explique les changements et les directions à prendre et c'est vous qui regardez sur le plan”. Elle a une copine qui a tout de suite pigé le truc mais elle, pas du tout, tu vois ? Et justement, à un moment donné, moi j'ai pas regardé, elle était derrière et on l'a perdue. Dans le métro, à République. On est arrivées sur le quai et les copines ont dit : "Elle est où Lola?" Panique, je suis retournée au carrefour, je l'ai retrouvée, il y avait un monsieur très gentil qui était avec elle, il commençait à m'appeler parce qu'elle lui avait donné le numéro, elle était en larmes... » (Chargée de projet dans une association, Paris. Une fille de 11 ans)

Une fois les parents rassurés par les premiers trajets effectués seuls par les enfants, les réseaux de transports en commun deviennent un élément structurant de la découverte et de l'exploration de la ville. Le long des lignes de bus et surtout de métro, les excursions hors de l'espace local s'articulent principalement autour d'espaces commerciaux (magasins de vêtements et de jeux, chaînes de restauration rapide), sportifs et culturels (principalement le cinéma). Les lieux de destination privilégiés par les enfants des enquêtés en dehors de l'espace local apparaissent davantage genrés à Paris qu'à Milan : les jeunes Parisiennes sortent en effet avant tout pour acheter des vêtements ou pour accompagner des amies en acheter, tandis que les garçons fréquentent principalement des boutiques de jeux de rôle, de jeux vidéo et d'articles de sport. Les sorties du quartier de ces derniers sont par ailleurs plus souvent liées à la pratique d'activités sportives, par exemple dans des skateparks situés dans d'autres arrondissements. Un pôle d'excursions féminines se dessine entre Châtelet et Les Halles, dont le quartier de Bastille serait le pendant masculin. À Milan en revanche, seules deux destinations sont évoquées par les parents, sans qu'aucune différence significative liée au sexe des enfants n'émerge : la découverte de la grande ville se cristallise autour du Corso Buenos Aires, avenue commerçante relativement proche du terrain d'enquête, et surtout autour de la cathédrale (Duomo) dans le centre historique de la ville.

Rarement atteinte par les enfants de la tranche d'âge qui nous intéresse, la dernière marche de l'autonomisation est constituée par les sorties nocturnes. Le sentiment de protection associé à l'espace local s'étiole avec le passage du jour à la nuit, qui réduit drastiquement le périmètre d'autonomie concédé : la nuit tend à être associée au vide, aux inconnus et aux marginaux (Melbin, 1978), ce qui explique la variation des horaires de retour au domicile en fonction de la saison.

Mère : Je pense que la journée ça ne pose aucun problème de le laisser se déplacer tout seul. Mais le soir, en revanche, je n'ai plus confiance.

Chercheur : À partir de quelle heure?

Mère : Disons que, bon, la limite correspond plus ou moins à la lumière.

(Cadre de banque, femme, Milan. Deux fils de 12 et 8 ans) 
« Il y a beaucoup de gens qui ne sont pas du tout fiables, qui sont complètement à côté de la plaque, et à Paris vous avez une faune naturelle qui s'éveille la nuit, qui n'est pas faite pour les enfants. » (Employée des postes, Paris. Deux fils de 13 et 11 ans, une fille de 8 ans)

Très peu d'enfants des enquêtés ont ainsi fait l'expérience de sorties nocturnes sans être accompagnés par un adulte au moins pour le trajet de retour, en dehors de quelques cas de déplacements effectués «à plusieurs », davantage rassurants pour les parents au-delà du seul cas des sorties nocturnes (Depeau, 2008 ; Rivière, 2014).

\section{ENCADREMENT PARENTAL ET DÉFINITION SOCIOLOGIQUE DU QUARTIER}

L'encadrement parental des pratiques urbaines des enfants revêt un caractère progressif, du domicile à la grande ville en passant par une gradation fine d'espaces que l'on a qualifiés de protégés. Ceux-ci se caractérisent par le fait que la circulation motorisée y est interdite, mais aussi par des formes de régulation collective des comportements des enfants et des tiers. En distinguant de manière analytique les étapes qui jalonnent l'encadrement parental, cet article confirme la pertinence d'une conception gradualiste des espaces publics, qui doivent être pensés sur le mode d'une hiérarchisation « fondée sur des niveaux d'accès à l'urbain du plus privé au plus public » (Dris, 2007). S'appuyant sur une enquête menée à la périphérie interne de deux métropoles européennes, il constitue une validation empirique de l'approche théorique élaborée par Lyn Lofland dans The public realm (Lofland, 1998). La distinction qu'elle y opère entre trois « domaines de la vie urbaine » (realms of city life) est de fait particulièrement opératoire du point de vue de l'étude des étapes de l'encadrement, qui vérifie la tripartition entre privé, local et public : d'abord centré autour du « monde du domicile et des réseaux de parenté et d'intimité » (private realm), le processus d'autonomisation se poursuit dans le « monde du voisinage et des réseaux d'interconnaissance » (parochial realm) avant de s'achever avec l'exploration autonome du « monde des étrangers et de la rue » (public realm).

Alors que Lofland pointe les risques qu'entraîne la confusion effectuée par de nombreuses recherches entre ces différents domaines, liée au fait qu'un même lieu peut ressortir de différents domaines selon le type d'interactions qui y domine provisoirement, les entretiens conduits auprès des parents à Paris et Milan montrent la nécessité de bien les distinguer, l'autonomie n'étant pleinement atteinte que lorsque les enfants sont autorisés à évoluer seuls dans chacun d'entre eux. Semblant faire sens aux yeux des enfants eux-mêmes (Harden, 2000), la distinction d'une sphère locale intermédiaire entre public et privé confirme que la vie dans les grandes villes tend à produire de la méfiance vis-à-vis des inconnus, mais n'implique pas la disparition des réseaux locaux d'interconnaissance et d'entraide (Fischer, 1981). Ainsi, si l'existence de 
normes de régulation de la présence des enfants dans les espaces publics transcende l'échelle locale, l'espace de résidence se voit attribuer un caractère spécifique par les parents, que le niveau plus élevé d'interconnaissance, y compris lorsque celle-ci n'est que superficielle (Blokland et Nast, 2014), tend à rassurer.

Relever la centralité de l'espace local dans l'encadrement parental conduit à inscrire nos observations dans la discussion relative à la définition sociologique du quartier. Celle-ci est « toujours problématique » (Grafmeyer, 2007), dans la mesure où l'identification des frontières du quartier varie sensiblement selon les habitants, en lien avec leurs propriétés sociales, leur ancienneté d'implantation ou encore leur position dans le cycle de vie (Authier, 2001b). Le rôle joué par l'interconnaissance localisée dans l'encadrement des pratiques urbaines des enfants invite à considérer que les limites du quartier vécu recoupent celles du réseau local d'interconnaissance d'ego : une telle définition compréhensive du quartier permet de mieux comprendre la récurrence de la figure du « village » dans la description que font les citadins de leur quartier, ainsi que les mécanismes de production de ses frontières par les acteurs. Elle aide aussi à mieux saisir les modalités du changement du rapport au quartier impliqué par la parentalité, en lien notamment avec la scolarisation des enfants, vecteur d'acquisition de capital social local (Weller et Bruegel, 2009). $\mathrm{Au}$-delà du cas des seuls parents, cette proposition de définition est susceptible d'éclairer la différenciation du rapport au quartier selon les propriétés sociales et les trajectoires des habitants, mais aussi selon les modalités de production de l'interconnaissance locale dans différents types de contextes urbains.

\section{RÉFÉRENCES BIBLIOGRAPHIQUES}

ARRIGONI, P. 2010. « Via Padova. Tra cosmopolis e ordine pubblico », dans M. Bricardi et P. Savoldi (sous la dir. de), Milano Downtown. Azione pubblica e luoghi dell'abitare, Milan, Et Al. Edizioni, p. 163-189.

AugÉ, M. 1986. Un ethnologue dans le métro, Paris, Hachette.

AUTHIER, J.-Y. (sous la dir. de) 2001a. Du domicile à la ville. Vivre en quartier ancien, Paris, Economica.

AUTHIER, J.-Y. 2001b. « Les rapports au quartier », dans J.-Y. Authier (sous la dir. de), Du domicile à la ville. Vivre en quartier ancien, Paris, Economica, p. 133-169.

AUTHIER, J.-Y.; LEHMAN-FRISCH, S. 2012. « Il était une fois... des enfants dans des quartiers gentrifiés à Paris et à San Francisco », Actes de la recherche en sciences sociales, $\mathrm{n}^{\circ} 195$, p. $58-73$.

Blokland, T. ; NAST, J. 2014. « From Public Familiarity to Confort Zone. The Relevance of Absent Ties for Belonging in Berlin's Mixed Neighbourhoods », International Journal of Urban and Regional Research, vol. 38, no 4, p. 1142-1159.

CAHILL, S. 1990. «Childhood and Public Life. Reaffirming Biographical Divisions », Social Problems, vol. 37, n 3, p. 390-402. 
DEPEAU, S. 2003. L'enfant en ville. Autonomie de déplacement et accessibilité environnementale, thèse de doctorat en psychologie, université Descartes-Paris 5.

DePeAU, S. 2008. "Radioscopie des territoires de la mobilité des enfants en milieu urbain. Comparaison entre Paris intra-muros et banlieue parisienne » [en ligne], Enfances, Familles, Générations, n 8, [URL : http://id.erudit.org/ iderudit/018489ar].

DRIS, N. 2007. « Les arrangements de visibilité dans les cheminements urbains. Du quartier proche au lointain dans la ville », dans M. Breviglieri et V. Cicchelli (sous la dir. de), Adolescences méditerranéeennes. L'espace public à petits pas, Paris, L'Harmattan, p. 63-76.

Fischer, C. 1981. « The Public and Private Worlds of City Life », American Sociological Review, vol. 46, n 3, p. 306-316.

GALSTER, G. 2001. « On the Nature of Neighborhood », Urban Studies, vol. 38, n 12, p. 2111-2124.

GLÉVAREC, H. 2010. "Les trois âges de la "culture de la chambre" », Ethnologie française, vol. 40, $\mathrm{n}^{\mathrm{0}} 1$, p. 19-30.

GRAFMEYER, Y. 2007. « Le quartier des sociologues », dans J.-Y. Authier, M.-H. Bacqué et F. Guérin-Pace (sous la dir. de), Le quartier. Enjeux scientifiques, actions politiques et pratiques sociales, Paris, La Découverte, p. 21-31.

GranOVETTER, M. 1973. « The Strength of Weak Ties », American Journal of Sociology, vol. 78, n 6, p. 1360-1380.

GRANIÉ M.-A. 2010. « Socialisation au risque et construction sociale des comportements de l'enfant piéton. Éléments de réflexion pour l'éducation routière », Enfances, Familles, Générations, n ${ }^{\circ} 12$, p. 88-110.

Harden, J. 2000. « There's No Place Like Home. The Public/Private Distinction in Children's Theorizing of Risk and Safety », Childhood, vol. 7, n 1, p. 43-59.

Holloway, S. ; Valentine, G. 2000. " Spatiality and the New Social Studies of Childhood », Sociology, vol. 34, no 4, p. 763-783.

HUGUENIN-RICHARD, F. 2010. « La mobilité des enfants à l'épreuve de la rue. Impacts de l'aménagement de zones 30 sur leurs comportements », Enfances, Familles, Générations, $\mathrm{n}^{\circ} 12$, p. 66-87.

JACOBS, J. 1961. The Death and Life of Great American Cities. The Failure of Town Planning, New York, Random House.

KARSten, L. ; VAN VliEt, W. 2006. « Children in the City. Reclaiming the Street », Children, Youth and Environments, vol. 16, $\mathrm{n}^{\mathrm{o}}$ 1, p. 151-167.

Lofland, L. 1973. A World of Strangers. Order and Action in Urban Public Space, New York, Basic Books.

Lofland, L. 1998. The Public Realm. Exploring the City's Quintessential Social Territory, New York, Aldine de Gruyter.

Melbin, M. 1978. « Night as Frontier», American Sociological Review, vol. 43, n 1, p. 3-22.

PAIN, R. 2006. « Paranoid Parenting? Rematerializing Risk and Fear for Children », Social and Cultural Geography, vol. 7, n 2, p. 221-243.

Prezza, M. ; Pilloni, S. ; Morabito, C. ; Sersante, C. ; Alparone, F. R. ; GiUliani, M. V. 2001. «The Influence of Psychosocial and Environmental 